\title{
Modeling of Acoustic Wave Absorption in Ocean
}

\author{
T. B. Mohite-Patil. \\ Lecturer (Sel.Gr.) \\ D.Y.Patil Engineering \\ College, \\ Kolhapur, India
}

\author{
A.K.Saran \\ Scientist \\ NIO, Goa, \\ India.
}

\author{
S. R. Sawant \\ Professor and Head \\ Shivaji University, \\ Kolhapur, India.
}

\author{
R. H.Chile \\ Professor \\ Guru Govind \\ College of Engg. \\ Nanded, India.
}

\author{
T. T. Mohite-Patil \\ Vice-Principal \\ Sanjeevan Engg \\ College, \\ Kolhapur, India.
}

\begin{abstract}
Effective under-water communication systems require detailed study of acoustic wave propagation in ocean. The preamplifier parameter tuning techniques for the underwater communication systems have been greatly affected by the recent information available on the respective area. Many investigators have studied the absorption of acoustic waves in ocean water and formulated empirical equations, however no one has made an attempt to offer the simulation model for the under-water acoustic propagation. This paper reports the comparative study of acoustic wave absorption carried out by means of modeling in MATLAB. The results of simulation have been compared with the practically measured values in the Arabian Sea near Goa and Atlantic Ocean. The model has been used to determine sound absorption for given values of depth (D), salinity (S), temperature $(\mathrm{T}), \mathrm{pH}$, and acoustic wave transmitter frequency (f). From the results a correction factor for faithful reception has been evaluated. Using the correction factor, magnitude of the received signal has been corrected.
\end{abstract}

\section{Keywords}

Sound absorption simulation model; Salinity; Temperature; Pressure; Sound Speed; Depth; Frequency; Mathematical formulae.

\section{INTRODUCTION}

The absorption of sound waves in sea water has been studied by many investigators ${ }^{[1-4]}$. Some researchers have carried out these studies using the measurements made by mixing the various ingredients present in sea water while others have used the measurements actually taken in the sea water. The results so far reported through former studies suffer from errors. This may be because the mixing of different ingredients is not taking place in the required proportions. Empirical formulae have been developed by these investigators however no one has made an attempt to design the simulation model for the underwater acoustic propagation.In the present communication we report simulation model developed for the acoustic wave absorption. The results of the simulation model have been compared with the actual results. The agreement is found to be satisfactory.

\subsection{Sound absorption}

Fisher and Simmons ${ }^{[1]}$ have proposed the equation for the sound absorption in sea water as follows.

$$
\alpha=\frac{A_{1} P_{1} f_{1} f^{2}}{f_{1}^{2}+f^{2}}+\frac{A_{2} P_{2} f_{2} f^{2}}{f_{2}^{2}+f^{2}}+A_{3} P_{3} f^{2}
$$

Where alpha $(\alpha)$ is coefficient of absorption in sea water.The first term in above equation represents the sound absorption due to the Boric Acid. The second term gives the sound absorption due to magnesium sulfate. The third term indicates the absorption due to pure water. The contribution of sound absorption due to other chemical ingredients has been found to be negligible. The third term provides sound absorption because of pure water. Constants $\mathrm{P}_{1}, \mathrm{P}_{2}$ and $\mathrm{P}_{3}$ indicate effect of pressure. Frequency dependence is shown by frequencies $f_{1}$ and $f_{2}$. These are relaxation frequencies of Boric Acid and Magnesium sulfate, $f$ is the frequency of sound. Values of $A_{1}, A_{2}$ and $A_{3}$ depend on water properties such as temperature, salinity and $\mathrm{pH}$ of water.

\subsection{Review of sound absorption investigations}

Research work on the absorption of sound in sea water forms topic of interest for many researchers. The outcome of their efforts is the formulation of different empirical relations for calculating the sound absorption in sea water as a function of different sound frequency ranges, different sound speed ranges and other ingredients of the sea water. Pinkerton ${ }^{[2]}$ using radar pulses of $7-66 \mathrm{MHz}$ frequencies has measured the absorption of sound in pure water. Leonard $^{[3]}$ at el have measured the absorption sound in pure water at frequencies of $15-480 \mathrm{KHz}$ using resonance method. Leonard et al. ${ }^{[4,6]}$ have established that the presence of $\mathrm{MgSO}_{4}$ in sea water is the cause of increase in sound absorption. Marsh and Schulkin ${ }^{[5]}$ have also developed the equations for sound absorption. Del Grosso [7] has published the absorption tables to summarize the results of Kurtze and Tamm. Murphy et al., ${ }^{[8]}$ have studied the absorption of sound in natural sea water at frequencies of 60,142272 , and $467 \mathrm{KHz}$ and observed that their values are lower than the values determined by Del Grosso. Fisher 
${ }^{[9]}$ has established the dependence of pressure on the sound absorption using the laboratory measurements. Shulkin and Marsh ${ }^{[5]}$ adjusting the constants in the sound absorption equation based on Wilson- Leonard's data ${ }^{[6]}$, have devised the equations which are functions of Salinity, Temperature and Pressure.Fisher and Simmons ${ }^{[1]}$ have published sound absorption equation based on the measurements carried out by Lyman and Fleming ${ }^{[10]}$ in artificial sea water. R.E. Francois and G. R. Garrison ${ }^{[11]}$ have formulated the equation for the sound absorption in the frequency range $400 \mathrm{~Hz}$ to $1 \mathrm{MHz}$ which includes the contribution of Boric Acid, Magnesium Sulfate and Pure water. The results given by this equation are very close to the practical results. We have used this equation to develop the model of sound absorption.

\section{SIMULATION MODEL}

The main simulation model of sound absorption and correction is shown in fig. 1 .

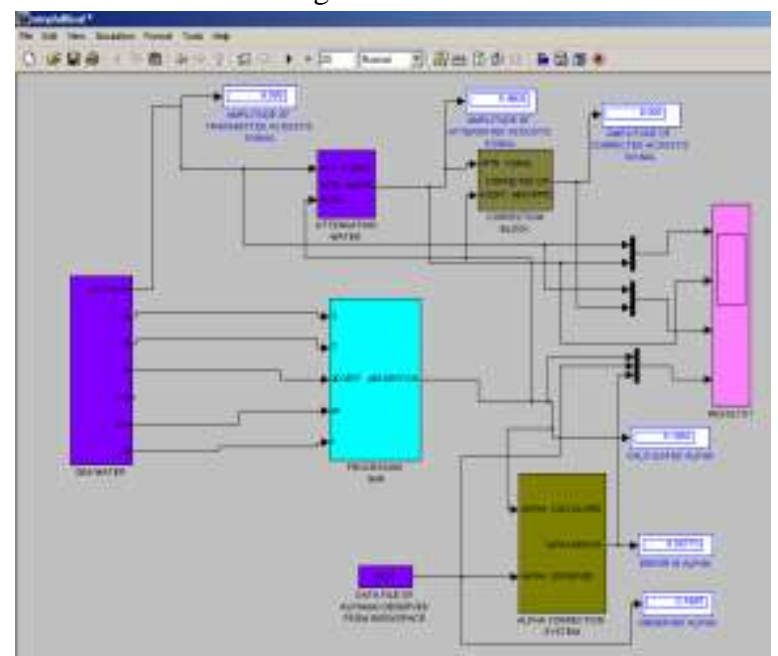

Fig.1. Main Simulation model for sound absorption and correction in sea water

It consists of five sub models namely

- $\quad$ Simin subsystem

Sea water parameter reading subsystem subsystem

Observed sound absorption coefficient

Sound absorption coefficient calculating subsystem

- Sound absorption coefficient correcting subsystem

- Acoustic Wave attenuating subsystem

- Attenuated acoustic signal correcting subsystem The simulation model reads the data concerned with sea water parameters such as temperature $(\mathrm{T})$, pressure $(\mathrm{P})$, salinity(S),depth(D), $\mathrm{pH}$ of sea water( $\mathrm{pH})$, frequency(f), sound speed (SS) from outside world with the Simin subsystem. Already measured data has been read with this subsystem. The prerecorded data imported in MATLAB workspace from excel file has been read by simin subsystem. The coefficient of sound absorption has been determined by using this data with the simulation model shown in fig. 2 .

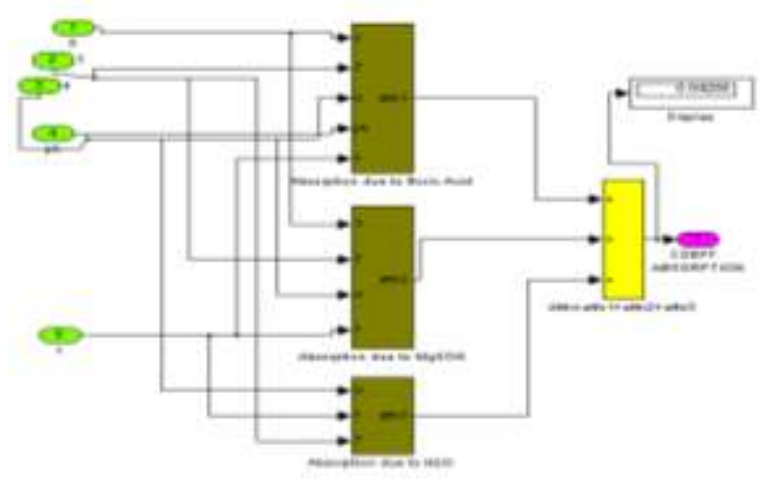

Fig.2 Simulation model for sound absorption

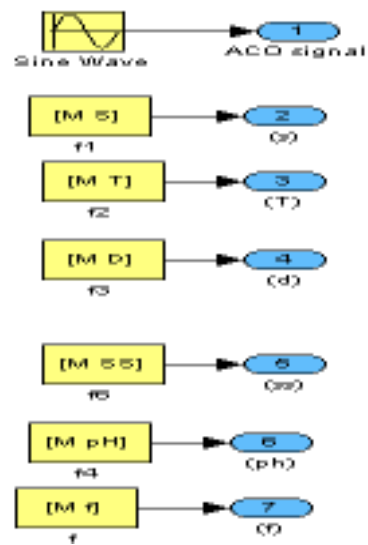

Fig.3. Reading external data for the Simulation model

Absorption coefficient due to Boric Acid

$\operatorname{attn}_{1}=\frac{A_{1} P_{1} f_{1} f^{2}}{f_{1}^{2}+f^{2}}$

$\mathrm{A}_{1}=\frac{8.86}{\mathrm{c}} \times 10^{(0.78 \mathrm{pH}-5)}$,

$\mathrm{dB} \mathrm{Km}{ }^{-1} \mathrm{KHz}^{-1}$

$\mathrm{P}_{1}=1$,

$\mathrm{f}_{1}=2.8(\mathrm{~S} / 35)^{0.5} \times 10^{(4-1245 / \theta)}$,

$\mathrm{KHz}$

Where $\mathrm{c}$ is the sound speed $(\mathrm{m} / \mathrm{s})$, given by $c=1412+3.21 \mathrm{~T}+1.19 \mathrm{~S}+0.0167 \mathrm{D}$,

$\mathrm{T}$ is the temperature $\left({ }^{0} \mathrm{C}\right)$,

$\theta=273+\mathrm{T}$,

$\mathrm{S}$ is the salinity $(\%)$, and $\mathrm{D}$ is the depth (m). 
Absorption coefficient due to $\mathrm{MgSO}_{4}$

$$
\begin{aligned}
& \text { attn }_{2}=\frac{A_{2} P_{2} f_{2} f^{2}}{f_{2}^{2}+f^{2}} \\
& \mathrm{~A}_{2}=21.44 \frac{\mathrm{S}}{\mathrm{c}}(1+0.025 \mathrm{~T}) \\
& \mathrm{P}_{2}=1-1.37 \times 10^{-4} \mathrm{D}+6.2 \times 10^{-9} \mathrm{D}^{2} \\
& \mathrm{f}_{2}=\frac{8.17 \times 10^{(8-1990 / \theta)}}{1+0.0018(\mathrm{~S}-35)} \\
& \text { Absorption coefficient due to Pure Water } \\
& \text { attn } 3=A_{3} P_{3} f^{2} \\
& \text { For } \mathrm{T} \leq 20^{0} \mathrm{C}, \\
& \mathrm{A}_{3}=4.937 \times 10^{-4}-2.59 \times 10^{-5} \mathrm{THz}+9.11 \times 10^{-1} \mathrm{~T}^{2}-1.50 \times 10^{-8} \mathrm{~T}^{3} \mathrm{~dB} \mathrm{Km} \mathrm{KHz}^{-2} \\
& \text { For T } \succ 20^{0} \mathrm{C}, \\
& \mathrm{A}_{3}=3.964 \times 10^{-4}-1.146 \times 10^{-5} \mathrm{~T}+1.45 \times 10^{-7} \mathrm{~T}^{2}-6.5 \times 10^{-10} \mathrm{~T}^{3} \mathrm{~dB} \mathrm{Km} \mathrm{KHz}^{-2} \\
& \mathrm{P}_{3}=1-3.83 \times 10^{-5} \mathrm{D}+4.9 \times 10^{-10} \mathrm{D}^{2}
\end{aligned}
$$

The operation of this model is based on the R.E.Francois and G.R. Garrison empirical formula for calculating the coefficient of sound absorption. From equation 1 it is seen that three factors contribute for the absorption of sound waves in sea water namely Boric Acid, Magnesium sulfate and pure water. The absorption coefficient has been determined with the simulation model as shown in fig 2 taking into account these three factors. The input data files have been read from workspace of MATLAB with simin submodel as shown in fig 3 In detail design of Boric Acid contribution based simulation model is as in figure 4 . The functioning of this model is based on the contribution of Boric Acid to absorption of the acoustic wave. Similarly absorptions due to the Magnesium Sulfate $\left(\mathrm{MgSO}_{4}\right)$ and pure water have been determined by the simulation models shown in fig (5) and fig (6). These models are based on the formulae as follows. The sound absorption model shown in figure. 2 is main model which calculates the coefficient of absorption of sound. The basic data like salinity, temperature, ph, depth and frequency to calculate the sound absorption is read from the input ports shown by numbers ranging from 1 to 6 . The coefficient of absorption due to Boric acid, due to magnesium sulpate and pure water are estimated by three subsystems and resultant is obtained by adding those three coefficients with a sim block adder.Error in calculated sound absorption coefficient and observed sound absorption coefficient has been determined with the simulation model as shown in fig 9. The required data to calculate the coefficient for absorption of sound in sea water is read from the excel files with the help of the simin blocks as shown in fig. 3. This data is output to the subsystems which do actual calculations for the coefficient of absorption. The schematic of simulation model designed to calculate the sound absorption due to boric acid is as shown in figure 4 . It consists of variety of mathematical operation blocks like multipliers, adders, dividers, power blocks and constant blocks. With these basic mathematical blocks the value of sound absorption due to boric acid is estimated for the formulae given by Fisher and Simmons. The sound speed is estimated by using the formula given by R.E. Francois and G. R. Garrison. This calculated sound speed is used to calculate the coefficient of absorption. The other terms like $\mathrm{A}_{1}, \mathrm{P}_{1}, \mathrm{f}_{1}$ and $\theta$ (degree kelvin) are also estimated with this model. The resultant coefficient of absorption due to boric acid is outputted on output port1 named as attn1.

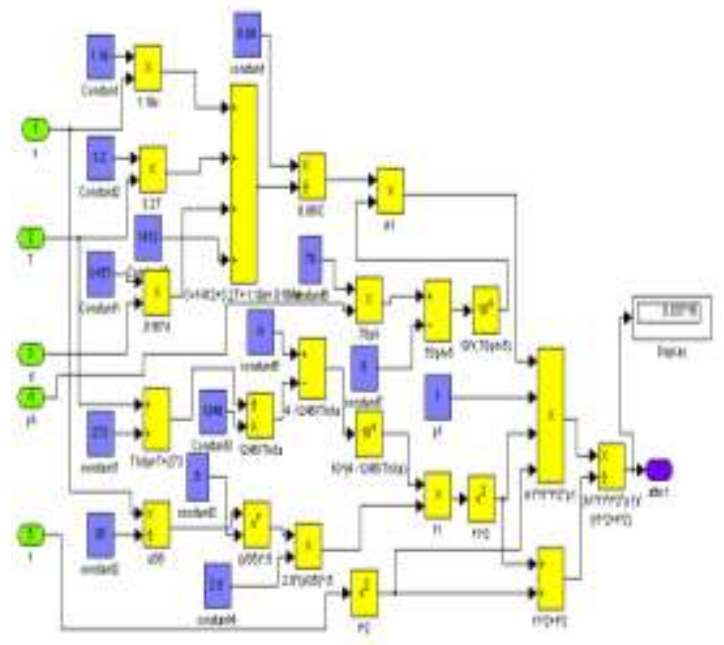

Fig.4. Simulation model of sound absorption due to Boric Acid in sea water.

The sound absorption due to magnesium sulphate in sea water is estimated with the model shown in fig. 5. This model is designed by using different blocks like input port block (through which the data concerned with the different parameters of sea water like salinity, temperature and depth), adder, multiplier, and divider, exponential and constant block. With these basic blocks the basic terms $\mathrm{A}_{2}$, $\mathrm{P}_{2}$, and $\mathrm{f}_{2}$ are used to calculate the coefficient of absorption due to magnesium sulphate and it is output on output port1 named attn2.The model shown in fig. 6 is designed using basic blocks like input port, adder, multiplier, exponential output port and constant block. This model estimates the terms $\mathrm{A}_{3}, \mathrm{P}_{3}$ and calculates the coefficient of absorption due to pure water. This value is output on output port1 named as attn3. 


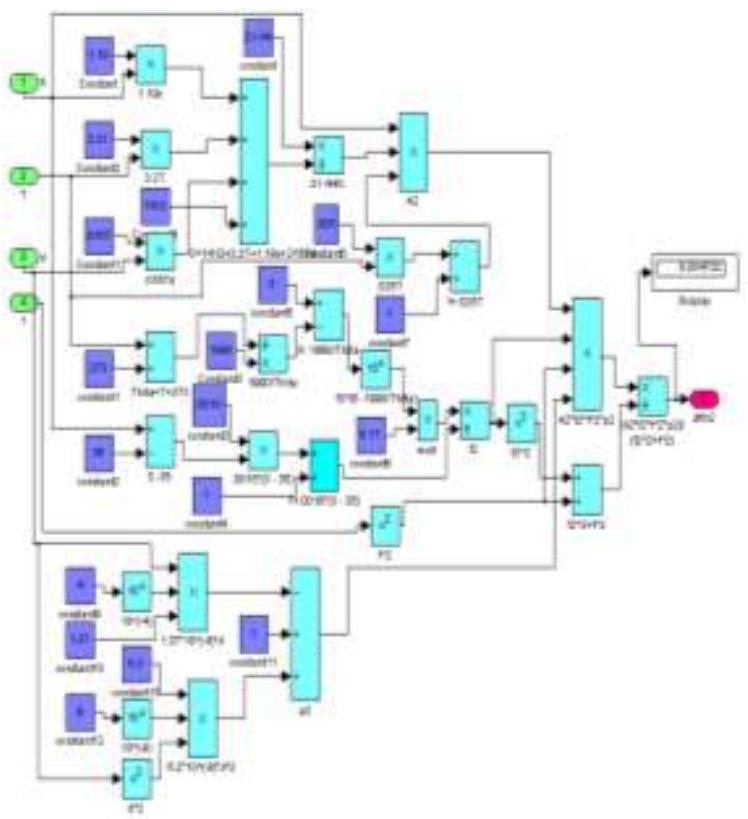

Fig.5. Simulation model of sound absorption due to Magnesium Sulfate in sea water.

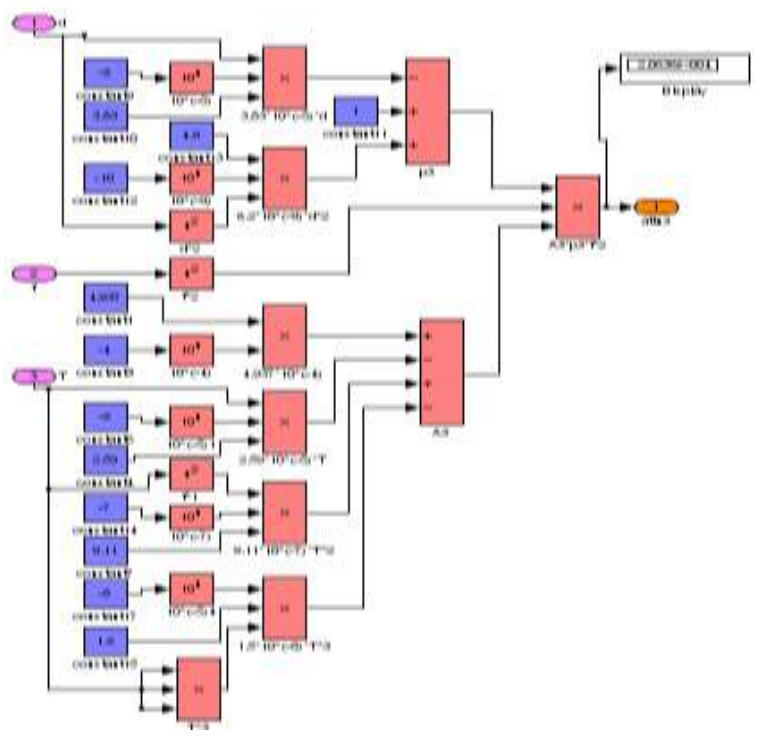

Fig.6. Simulation model of sound absorption (attenuation) due to Pure Water.

The model shown in fig. 7 generates the attenuated acoustic signal artificially using blocks like input port, multiplier and output port. The attenuation provided to the acoustic wave propagating through sea water is totally governed by the resultant coefficient of absorption of the sea water. The multiplier block multiplies the transmitted acoustic signal with the resultant coefficient of absorption and gives artificially attenuated acoustic signal to the output port1 named as ATTN water.

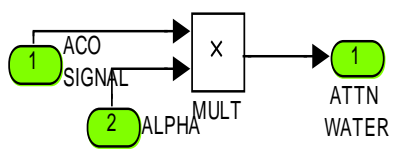

Fig.7. Simulation model of sound correction

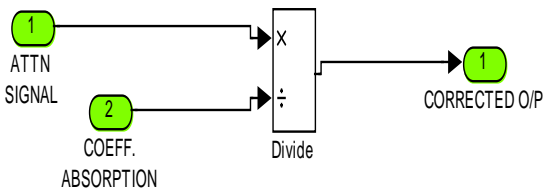

Fig.8. Simulation model of sound wave absorption (attenuation) factor correction model.

The model in fig. 8 is named as correction model which consists of input port block, divide and output port. Through input port block 1 the attenuated signal is read. The resultant coefficient of absorption estimated by the different models explained earlier is read through input port 2 . With divide block the attenuated acoustic signal is corrected to input propagated signal and put on output port 1 named as corrected output.

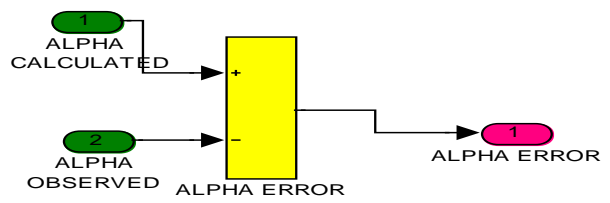

Fig.9. Sound absorption error detecting simulation model

Error in calculated sound absorption coefficient and observed sound absorption coefficient has been determined with the simulation model as shown in fig 9. with two input ports named as alpha calculated and alpha observed the data concerned with calculated coefficient of absorption and the observed coefficient of absorption is read and an error in alpha is calculated with subtract block.

\section{RESULTS}

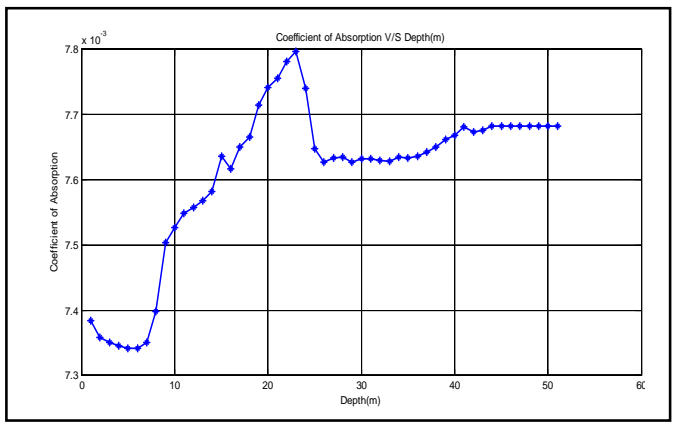


Fig.10. coefficient of absorption V/S Depth

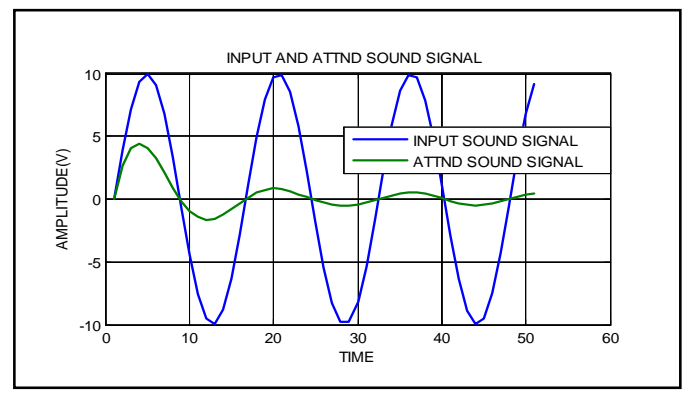

Fig.11. The transmitted acoustic signal and attenuated signal

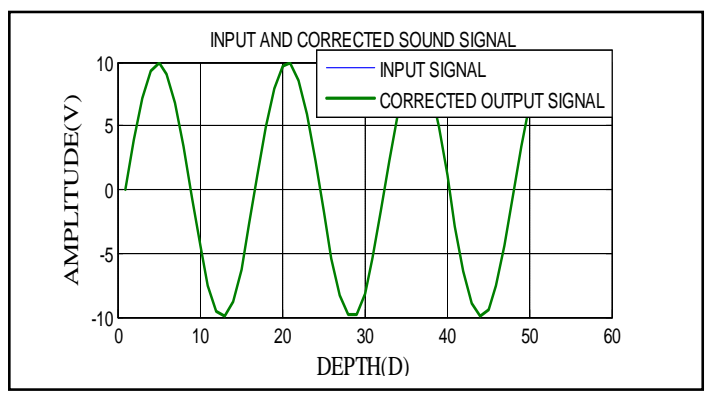

Fig.12. Corrected output acoustic signal

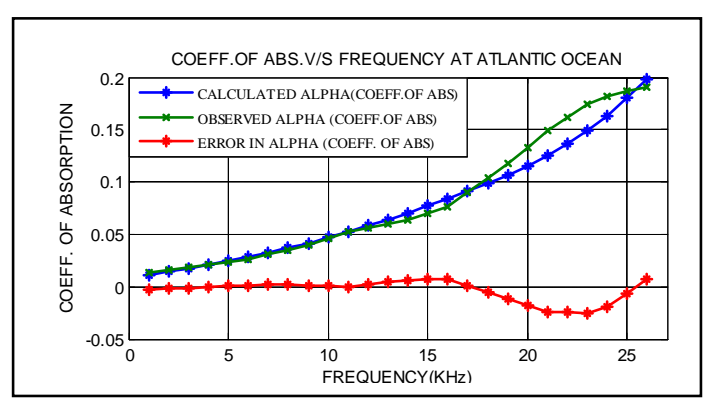

Fig.13. Simulation result for data at Atlantic Ocean

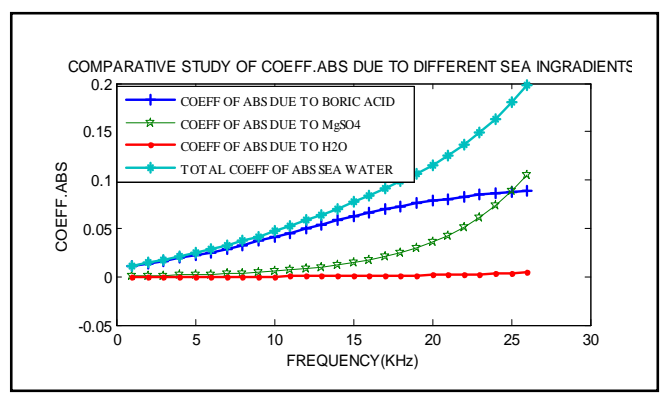

Fig.14. Coefficient of absorption due different parameters

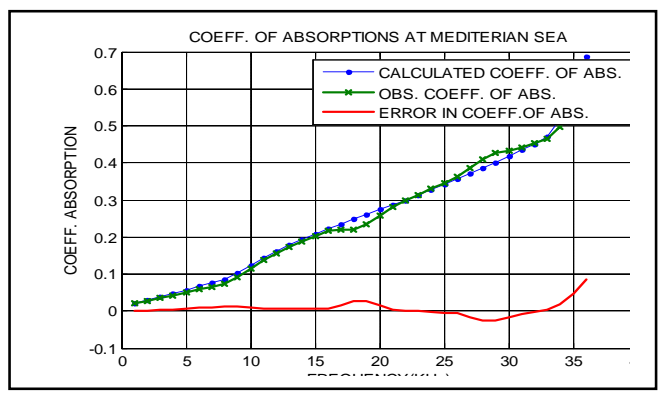

Fig.15. Simulation result for data at Mediterian Sea

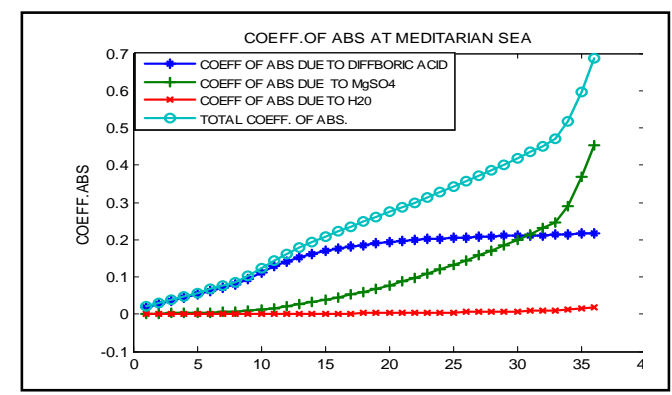

Fig.16. Coefficient of absorption due to different parameters

\subsection{Comments on graph}

Figure.10. represents variation of total absorption of sound waves as a function of depth of sea for the practical data collected at Goa. It is seen that the total absorption is maximum $\left(7.8 \times 10^{-3} \mathrm{~dB} \mathrm{Km}^{-1}\right)$ at about $220 \mathrm{~m}$ distance from the sea surface. The transmitted acoustic signal and the attenuated acoustic signal are shown in fig.11 and fig.12. The simulation results are shown in fig.13 and fig.15 where in the calculated alpha and simulated alphas have been compared and the error has been calculated. The error observed is negligibly small. Also individual contribution of sea parameters is also shown in fig.14 and fig. 16 .

\section{CONCLUSION}

With the present simulation model coefficient of absorption has been calculated by inputting the sea water parameters. Using these absorption coefficients the simulation model corrects the received acoustic signal. In this manner the simulation model proposed helps provide faithful reception in the underwater communication irrespective of the sea and water quality.

\section{ACKNOWLEDGEMENTS}

The authors are thankful to the National Institute of Oceanography (NIO) Goa, India for providing the practical data of whole year of Arabian Sea water. 


\section{REFERENCES}

[1] F.H.Fisher and V.P.Simmons, "Sound absorption in sea water,” J. Acoustic. Soc. Am.62, 558-564(1977).

[2] J.M.M .Pinkerton, "A pulse method for the measurement of ultrasonic absorption in liquids: results for water," Nature 160,128(1947).

[3] R. W. Leonard, "The attenuation of ultrasonic waves in water", J.A coust. Soc. Am. 20, 224(1948).

[4] R.W. Leonard, P.C. Combs, and L.R. Skidmore, "The attenuation of sound in synthetic sea water," J.Acoustic. Soc. Am.21, 63(1949).

[5] H. W. Marsh and M. Schulkin, "Report on the status of Project AMOS Tch. Memo. No.1110-023-52, U.S.Navy Underwater Sound Lab., New London, CT (1952).

[6] O. B. Wilson, Jr., R. W. Leonard, "Measurement of sound absorption in aqueous salt solutions by a resonator method,"J. Acoustic. Soc. Am. 26, 223226(1954)
[7] V.A. Del Grosso, "Dependence of sound absorption on concentration, frequency, and temperature in $\mathrm{MgSO}_{4}$ solutions equivalent to sea water" Naval Res. Lab., Washington, DC (January 1954).

[8] S. R. Murphy, G. R. Garrison and D. S. Potter,"Sound absorption at 50 to $500 \mathrm{kc}$ from transmission measurements in the sea," J.Acoust. Soc. Am.30, $871-875(1958)$.

[9] F. H. Fisher, "Effect of high pressure on sound absorption and chemical equilibrium,"J.Acoust.Soc.Am.30,442 - 448(1958).

[10] J. Lyman, R. H. Fleming, "Composition of sea water,'J.Mar.Res.3,134-146(1940).

[11] R.E.Francois and G.R. Garrison,"Sound absorption based on ocean measurements.PartII:Boric acid contribution and equation for total absorption," J. Acoustic. Soc. Am.72(6),1879-1890(1982). 